\title{
Actual tendencies of Hallux Valgus surgical treatment
}

\author{
Julian Ruci *, Edvin Selmani
}

Received: 10 November 2021 / Accepted: 04 December 2021 / Published online: 20 January 2022

This article is published with open access at https://journal.astes.org.al

(C) The author(s) 2022. \& Copyright (C) 2022, the Albanian Society for Trauma and Emergency Surgery

(c) The Albanian Journal of Trauma and Emergency Surgery is an Open Access Journal. All articles are distributed under the terms of the Creative Commons Attribution Non-Commercial License: http://creativecommons.org/licenses/by-nc/4.0/) which permits unrestricted non-commercial use, distribution, and reproduction in any medium provided the original work is properly cited.

\begin{abstract}
The purpose of this study is to evaluate which of the methods selected in patients with moderate or severe hallux valgus a result in a better correction of hallux valgus angle (HVA) and intermetatarsal angle (IMA) in Scarf osteotomy as compared to Chevron osteotomy.

Material and methods; In our study, we selected 36 patients, 16 scarf and 20 chevron osteotomies, with all surgical options from skin incision, capsular and bunionectomy to bone reorientation. Deformities of patients were classified as mild, moderate and severe according to clinical and radiological findings. The results were measured using radiographic HVA, IMA and distal metatarsal articular angle (DMAA).

Results: No statistical differences were found in HVA, IMA and DMAA between scarf and chevron osteotomy in mild to moderate hallux valgus. In severe hallux valgus, Scarf osteotomy corrected HVA better than Chevron, although this group consisted of twelve patients. Two patients in the Chevron group and three in the Scarf group developed subluxation of the metatarsophalangeal joint.

Conclusion: In patients with moderate and severe hallux valgus the results of Scarf and Chevron osteotomy have no specific difference. Change to IMA angle with the subluxation of the first metatarsophalangeal joint some months after operation were the main cause for insufficient correction. We favor the Scarf osteotomy because it is more profitable, with correction of HVA and IMA. Keywords; hallux valgus, bunionectomy, osteotomy, metatarsophalangeal joint.
\end{abstract}

\section{Abbreviations}

Hallux Valgus Angle - HVA; Intermetatarsal Angle IMA; Distal Metatarsal Articular Angle - DMAA; First Metatarsophalangeal Joint - MTPJ1; American Orthopaedic Foot and Ankle Society - AOFAS; Distal Soft Tissue Procedure - DSTP; First Metatars - MT1; Complex Regional Pain Syndrome - CRPS;

\section{Introduction:}

Scarf osteotomy is a widely accepted osteotomy for correction of hallux valgus, after the '90. The SCARF osteotomy has become a widely used procedure in middle Europe since the introduction and the development of specially designed osteosyntheses material.

Original article, no submission or publication in advance or in parallel

* Corresponding author:

Julian Ruci, MD,

* julian.ruci@yahoo.com

1 University Hospital of Trauma, Tirana, ALBANIA.
Proximal and distal metatarsal osteotomies allow correction of higher intermetatarsal angle (IMA).

Chevron osteotomy is modern procedure also, that should be able to correct higher IMA with the advantage of intrinsic stability. Radiological assessment is based in hallux valgus angle (HVA), intermetatarsal angle (Fig.1)and distal metatarsal angle (DMAA) [1, 2, 3].

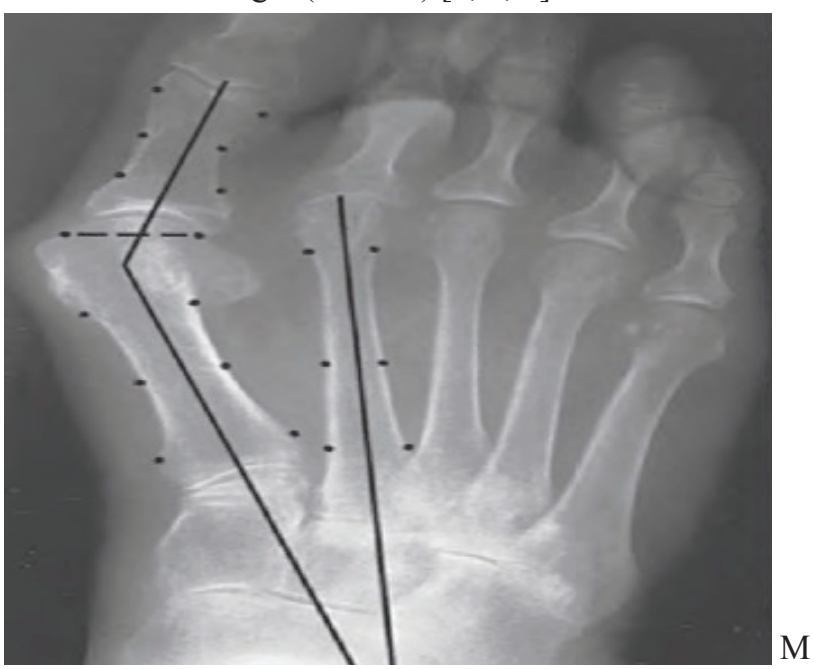

Figure 1 Foot A/P view with Hallux Valgus deformation 
These radiological parameters are used with different cut-off points in algorithms to choose between distal, shaft and proximal osteotomy. IMA points are used in the range between 11-20 degrees, for classification in mild, moderate and severe hallux valgus. However, no statistical difference was demonstrated in correction of IMA and HVA in randomized controlled trials comparing tarsometatarsal arthrodesis versus distal osteotomy and distal versus shaft osteotomy. These studies were performed in hallux valgus groups where mild deformities were included. The validity of IMA in preoperative planning should be assessed.

Mild deformities might influence our results, we did need a bigger group in order to test differences between scarf and chevron osteotomy in subgroups $[1,6]$. Algorithms for more aggressive surgery are justified in moderate and severe hallux valgus deformities to obtain better correction of HVA. However, it is recognized that clinical and radiological parameters are lacking in evaluating operative results.

In spite of this, radiological and clinical parameters are used in daily practice to consider more aggressive surgery in order to obtain better correction.

Radiological parameters are used in our study to evaluate whether in patients with moderate or severe hallux valgus Scarf osteotomy results in a better correction of HVA and IMA, as compared to Chevron osteotomy [7]. The results will be compared according to preoperative IMA
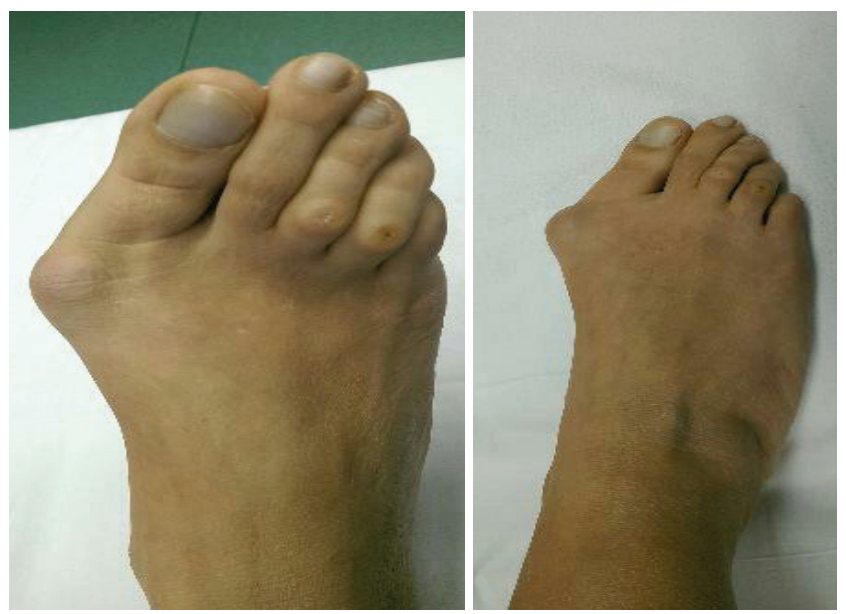

Figure 2 Hallux Valgus deformation

\section{Materials and Methods:}

The period of the survey was from December 2010 to July 2015 with 36 patients, 3 bilateral cases,included in the study. 20 Chevron osteotomies and 16 Scarf osteotomies were seen at follow-up after 3 years after surgery. Preoperative IMA was used to define patient groups; mild hallux valgus was defined with IMA of 11-16 degrees, moderate hallux valgus was defined with IMA from 16 to 20 degrees, and severe hallux valgus was defined with IMA from 20 degrees or more.(Fig. 2)

Clinical assessment procedures, radiological data retrieval, was prepared according to American Orthopaedic
Foot and Ankle Society scores. Operative procedures and postoperative treatment are described in details as follows. Radiological evaluation was performed according to standardized procedures,with standard dorsoplantar and lateral foot $\mathrm{x}$-ray with weight bearing. The angle between the first and second metatarsals is between 8 to 9 degrees, usually considered to be the upper limits of normal.

The valgus angle of the first metatarsophalangeal joint also is more than 15 to 20 degrees, considered to be the upper limits of normal weight bearing $[1,2,5,9]$.

HVA was measured as the angle between the line connecting the centre of the base to the centre of the head of MT1 and the line connecting the midpoints of the proximal and distal articular surfaces of the proximal phalanx. The IMA is defined as the angle between the longitudinal axis of MT1 and the line bisecting the distal and proximal diaphyseal portions of MT.

The distal metatarsal articular angle (DMAA) was measured according to standard guidelines. Markers were placed at the most medial and most lateral extent of the metatarsal articular surface. A line was drawn connecting the two markers. Another line was drawn perpendicular to this line, according to the literature.

We classified subluxation of the first MTP joint if the lateral articular border of the proximal phalanx passes the lateral articular border of the middle phalanx.

Operative procedures: Operations were performed using spinal locoregional anestesia or nerve block anesthesia. Also a tourniquet just above the knee joint was used. (fig. 3)

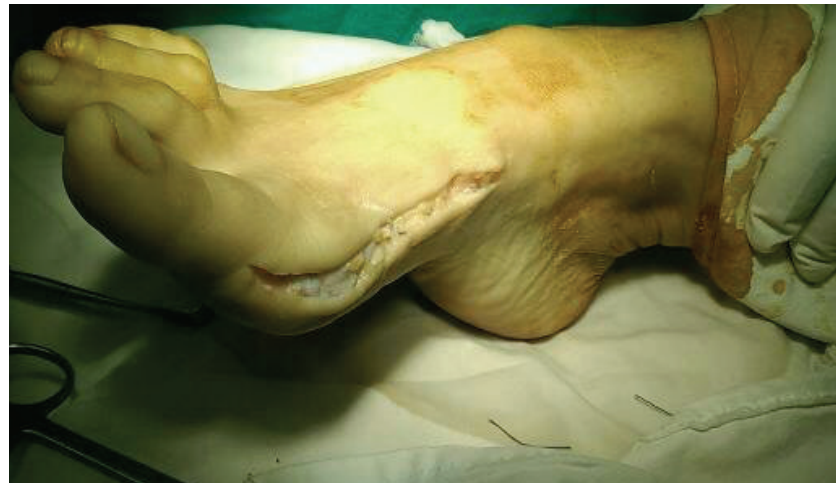

Figure 3 Type of incision in Hallux Valgus deformation

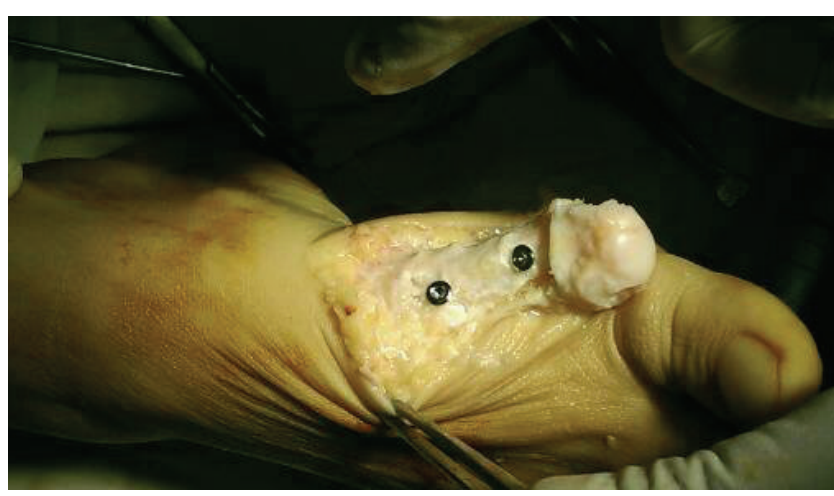

Figure 4 Visualization of metatarsal joint. 
The standard approach for Scarf osteotomy was via a medial incision, at the junction of the plantar and the dorsal skin,with its proximal part below the surface projection of the metatarse $[1,2,4]$. The joint capsule and the medial collateral ligament of the MPI joint were incised horizontally, at the line of the first incision. The medial aspect of the metatarsal head was exposed. (Fig. 4) The medial eminence of the metatarsal head was partially resected.

From an additional small dorsal interdigital approach, the lateral capsule was released longitudinally above the lateral sesamoid, leaving the plantar plate and the adductor tendon intact.

The sesamoids were then mobilized trying to turn them back to the anatomic position. In preparation for the three osteotomy cuts, two guiding 1.2-mm K wires were inserted at the corner points of the planned SCARF cut as entry point of the proximal pin averaged $1 \mathrm{~cm}$ distal to the first metatarsal medial cuneiform joint line, over the concavity of the inferior aspect of the metatarsal at the junction of the plantarinferior to the medial aspect.

The entrance point of the distal pin crossed the metatarsal head $5 \mathrm{~mm}$ proximal to the dorsal cartilage surface in the dorsal to the medial aspect. The translation is greater for larger IM angles. For maximal stability of the osteotomy after translation, it is necessary to orient the proximal and distal osteotomy cut strictly parallel to each other.

Translation and lowering was indicated for hallux valgus with intermittent metatarsalgia or a deficit of the first metatarsal head in weight-bearing. This is best diagnosed clinically. The osteotomy was modified by directing the orientation of the $\mathrm{K}$ wires.

Translation andshortening of the first metatarsal could be performed.Shortening was obtained by increasing the obliquity of the anterior and posterior cuts to the longitudinal axis of the second metatarsal. Additional shortening was indicated in severe forefoot deformity with luxation of the lesser toes at the metatarso-phalangeal joints $[1,2]$.

Translation and lengthening were indicated in cases with short first metatarsal combined with metatarsalgia.

Fixation of the osteotomy was achieved using two small cannulated bicortical compressive screw of steel or titanium material. (fig. 5) A medial capsulorraphy was performed to secure soft-tissue realignment

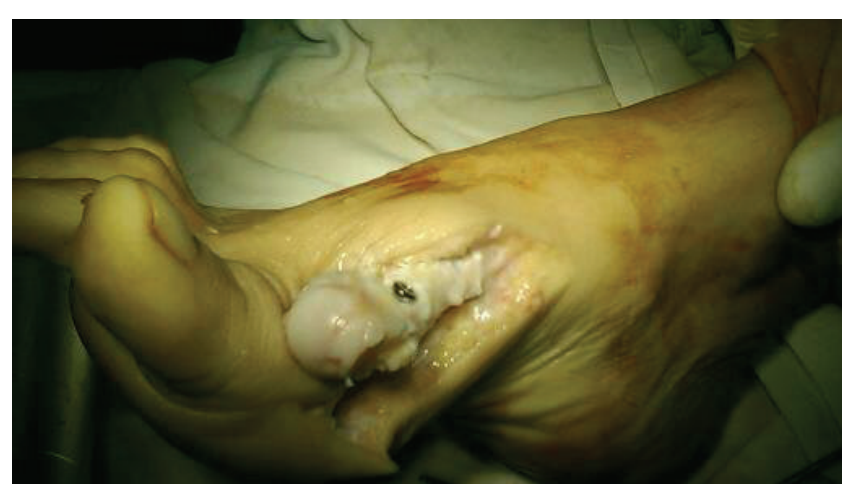

Figure 5. Cannulated bicortical compressive screw
The chevron osteotomy which was described first by Corless in 1976 as a modification of the Mitchell procedure to correct the bunion associated with mild-to-moderate metatarsus primus varus. The procedure consists of two parts: correction of metatarsus primus varus by a V-shaped osteotomy in the sagittal plane through the metatarsal head and neck, followed by lateral shifting of the metatarsal head and trimming of the proximal fragment.

It was performed through a medial incision centred over the first metatarsophalangeal joint (MTPJ1) $[6,7]$.

The dorso-medial cutaneus nerve was protected. The medial capsule was opened longitudinally and a strip of capsule was excised. The medial eminence of the first metatarsal head was removed. A transarticular transverse lateral release was performed until the proximal phalanx was in line with MT1.

A 60- degree V-osteotomy centred in the first metatarsal head was performed; the capitafragment was displaced laterally. The osteotomy was secured provisory with a percutaneous Kwire After completing the osteotomy, the distal fragment was displaced laterally to reduce the intermetatarsal angle.

Lateral displacement was achieved by pushing the distal fragment laterally while holding the proximal fragment of the first metatarsal in place. The final osteosinthesis was achieved using a cortical steel or titanium screw.

Postoperative complications include: Subluxated joint, Avascular necrosis, skin infection, nonunion [9].

\section{Results:}

There was significant improvement in correction of the HVA and IMA in Scarf and Chevron osteotomies in patients with mild, moderate and severe hallux valgus. In mild and moderate hallux valgus, pre- and postoperative improvement of HVA and IMA between scarf and chevron osteotomy differences did not reach statistical significance. The severe hallux valgus group consisted of twelve patients; the statistically significant clinical difference was reached in favour of the Chevron osteotomy in postoperative HVA and correction of HVA. Scarf osteotomy resulted in better correction of IMA; this finding was not statistically significant.

All 36 cases in moderate and severe hallux valgus patients had non-congruent joints preoperative. The cases with preoperative congruent joints were not overcorrected. Of 36 cases, eight cases developed recurrence of hallux valgus, with an average HVA of 31 degrees, because of dislocation of the MTPJ1 after initial adequate correction.

Three of these patients were in the Chevron group and all were preoperative classified as moderate hallux valgus. Five of these patients were in the Scarf group, two cases, one of them,71 years old, had preoperative severe and five moderate hallux valgus. The remaining 90 percent of the moderate and severe group had an average postoperative HVA of 17 degrees with congruent joints. At the patients with recurrence was proposed revision surgery, which they 
were not inclined to undergo because an clinical acceptable reduction of pain after the first operative intervention was reached.

As seen in AOFAS score no differences between Scarf and Chevron osteotomy were found. In comparison with other studies the number of patients with increased pain with Scarf osteotomy increased from four to seven patients, six of these were mild and one was moderate . Seven patients found that recovery after surgery was more quickly in the foot corrected by Chevron osteotomy, however, radiologically nor by AOFAS clinical differences could be found at the follow-up moments.

\section{Discussion:}

This study was made to evaluate patients with moderate or severe hallux valgus a Scarf osteotomy results in a better correction of HVA and IMA as compared to Chevron osteotomy. In patients with moderate and severe hallux valgus no differences were found between Scarf and Chevron osteotomy. In our study we found no differences between Scarf and Chevron osteotomy in correction of HVA and IMA in patients with moderate and severe hallux valgus. Our findings are consistent with these of previous randomized controlled trials. However, this is our first randomized trial to compare distal versus shaft osteotomy in patients with mild, moderate and severe hallux valgus. Patients with severe hallux valgus obtained a better correction with chevron osteotomy than patients who received a scarf osteotomy, although a group of twelve patients is too small for definitive conclusions. However, scarf osteotomy was expected to correct moderate and severe hallux valgus better $[3,8]$. Combined scarf osteotomy with open lateral release and Akin osteotomy is possible in selected cases [4]. These additional operative procedures might be a more important factor in obtaining results than the type of osteotomy. In cases with a wide metatarsal shaft, more translation could be achieved with the Scarf osteotomy; a wide subcapital fragment was seen more often, which allowed more translation in Chevron osteotomy.

In Scarf osteotomy rotation of the distal fragment is possible; this allows more correction of the distal fragment with a small increase in distal metatarsal articular angle. This had been performed in part of the moderate and severe cases. We could not find an influence of refining of technique in these cases. Clinical improvement between patients who received Scarf or Chevron osteotomy did not change. Recovery was between 6 week to 6 months. The extra soft tissue damage in the Scarf osteotomy seems small in comparison to the Chevron procedure. At follow-up no radiographic or clinical signical differences were found. More extensive surgery should be taken into consideration in preoperative planningif severe cases are contigent of treatment. In previous studies complications between both procedures were equal. Avascular necrosis occurred in the Chevron group in the early phase,as refered in other articles, were not seen $[6,7]$.
By refining our technique this complication could be intercepted by taking care of plantar vasculair blood supply. The lower incidence of complications is now in favour of the Chevron group. The IMA is acknowledged as an important factor in the prediction of the possible reduction of the hallux valgus deformity and is, therefore, used as a parameter for classification by algorithms. In patients with moderate and severe hallux valgus 90 percent good correction was achieved with Scarf and Chevron osteotomy. In the remaining ten percent of patients with moderate and severe hallux valgus recurrences occurred in ten percent, which corresponds with literature[2, 3, 5].

Pain reduction for these 12 patients was acceptable, more aggressive surgery seems indicated. Subluxation of the sesamoid on the plantar anterior X-ray might be an option, although other studies found it less reliable on X-rays because of rotational deformity. A possible explanation for secondary subluxation could be that transarticular release was insufficient. Than it is likely that the lateral sesamoid and the adductor hallucis were not completely released

Patients with moderate to severe hallux valgus and subluxation are usually corrected with shaft or proximal osteotomy including a distal soft tissue procedure (DSTP).

However, this concerns short-term follow-up, and patients with subluxated joints might predispose to artritis. It is clinically relevant to lower subluxation rate (19 percent in moderate severe hallux valgus in other studies), without exposing patients to excessive operative procedures (81 percent in the same group), because of possible complications like hallux varus and CRPS.

The other possibility for secondary subluxation could be that the biomechanical axis was insufficiently restored, because translation of the distal fragment in distal and shaft osteotomy was limited.Another reason was the insufficient osteosinthesis of the ostheotomy. Alignment of the first ray was restored after surgery, cases of recurrence occurred after three months follow-up.

Operative methods to improve correction the biomechanical axis are following; a proximal osteotomy does allow more translation and rotation of the distal fragment, although recurrences of hallux valgus from four percent and more are still described in these procedures. Another option would be to perform an Akin osteotomy to enhance alignment in the proximal phalanx. Knowledge and guidelines regarding hallux valgus correction surgery did improve quality of correction. In our study IMA was correlated with HVA outcome; however, cut-off points were less accurate to predict cases that develop recurrences.

Radiological parameters used in algorithms provide insight of factors to consider in preoperative planning of hallux valgus surgery. However, there is still a lack of evidence which cut-off point and operative procedure to choose. Operative strategy should provide an optimal balance between adequate correction and prevention of recurrences. Possible complications of more aggressive surgery should be considered carefully with their possible benefit in achieving better correction. 
A downside of more extensive foot procedures seems slow recovery because of increased swelling and pain. Further research is necessary to improve identification of patients that are predisposed to secondary subluxation. An optimal operative strategy should be evidence-based, provide information on which structures need to be released laterally, on the kind of osteotomy and on the necessity of an Akin osteotomy.

\section{Conclusion:}

In patients with moderate and severe hallux valgus the results of Scarf and Chevron osteotomy do not differ. Subluxation of the MTPJ1 was the main cause for insufficient correction. We favour the Chevron osteotomy because it is less invasive, without sacrificing correction of HVA and IMA. The rate of CRPS in the scarf osteotomy group did raise concern. The use of IMA to predict recurrences was limited.

\section{Declaration of Conflicting Interests and Ethics;}

The authors declare no conflict of interest. This research study complies with research publishing ethics. The scientific and legal responsibility for manuscripts published in Albanian Journal of Trauma and Emergency Surgery AJTES, belongs to the author(s).

Acknowledgements: None.

\section{References}

1. Barouk, LS: The first metatarsal Scarf osteotomy associated with the first phalanx osteotomy in the hallux valgus treatment. Extrait de medicine et chirurgie du pied. EFORT, Foot and ankle speciality day: 133-160, 1993

2. Barouk, LS: Scarf osteotomy of the first metatarsal in the treatment of hallux valgus. Foot Diseases 2(1):35-48, 1995

3. Coughlin, M. J.: Hallux valgus. Instr Course Lect, 46: 357-91, 1997

4. Mann, R. A.: Decision-making in bunion surgery. Instr Course Lect, 39: 3-13, 1990

5. Schneider, W.; Csepan, R.; and Knahr, K.: Reproducibility of the radiographic metatarsophalangeal angle in hallux surgery. J Bone Joint Surg Am, 85-A(3): 494-9, 2003

6. Trnka, H. J.; Zembsch, A.; Easley, M. E.; Salzer, M.; Ritschl, P.; and Myerson, M. S.:The chevron osteotomy for correction of hallux valgus. Comparison of findings after two and five years of follow-up. J Bone Joint Surg Am, 82-A(10): 1373-8, 2000.

7. Crosby, LA; Bozarth, GR: Fixation comparison for Chevron osteotomies. Foot \& Ankle Int'1, 19(1):41-43, 1998

8. Bonnel, F; Canovas, F; Poiree, G; et al: Evaluation of the Scarf osteotomy in hallux valgus related to distal metatarsal articular angle: a prospective study of 79 operated cases. Rev Chir

9. Orthop Reparatrice Appar Mot. July 85 (4):381-6, 1999

10. Meier, P. J., and Kenzora, J. E.: The risks and benefits of distal first metatarsal osteotomies. Foot Ankle, 6(1): 7-17, 1985. 